\title{
Hallazgo de restos arqueológicos en Chullkumayu, en la cuenca alta del río Pampas-Ayacucho
}

\author{
Finding of archaeological remains in Chullkumayu, in the upper basin of \\ the Pampas-Ayacucho river
}

\author{
Juan Conde Vilca \\ https://orcid.org/00oo-0002-5932-6481 \\ Universidad Nacional San Cristóbal de Huamanga \\ juan.conde.11@unsch.edu.pe
}

\section{INTRODUCCIÓN}

En el año 2012, durante la construcción de la carretera que conectaría las capitales de los distritos de Chuschi y Totos, dejaron al descubierto restos arqueológicos, específicamente en el lugar conocido por la población como Chullkumayu. Estos restos fueron hallados en las coordenadas UTM 565821 E, 8497275 N, a 2941 m.s.n.m., en la margen derecha de la subcuenca del río Chullkumayu, en la margen izquierda de la cuenca alta del río Pampas. Políticamente, se encuentra en la jurisdicción de la comunidad campesina de Quispillaccta, distrito de Chuschi, provincia de Cangallo y región de Ayacucho. Se encuentra a $4 \mathrm{~km}$ aproximadamente al Oeste de Quispillaccta, a $2 \mathrm{~km}$ aproximadamente al sureste de Pirhuamarka y a $3 \mathrm{~km}$ aproximadamente de Llacctahurán. 


\section{LOS MATERIALES ARQUEOLÓ- GICOS REGISTRADOS}

Lamentablemente, los restos arqueológicos fueron destruidos y no fueron registrados in situ por un especialista, es más, ni se tiene ninguna referencia de estas evidencias arqueológicas hasta la actualidad. A pesar de que fue disturbada y sufrió los daños irreparables, muchos de los materiales salieron ilesos o poco dañados; las cuales fueron recuperadas y trasladadas a la I. E. de Quispillaccta. La particularidad de los materiales reside en su estado de conservación, pues muchos materiales arqueológicos de este tipo no se conservan en la sierra peruana, por la misma condición ambiental.

Los primeros análisis de los materiales nos permitieron identificar que se trata de un entierro prehispánico; lamentablemente sin contexto arqueológico. A pesar de ella, es necesaria hacer las investigaciones

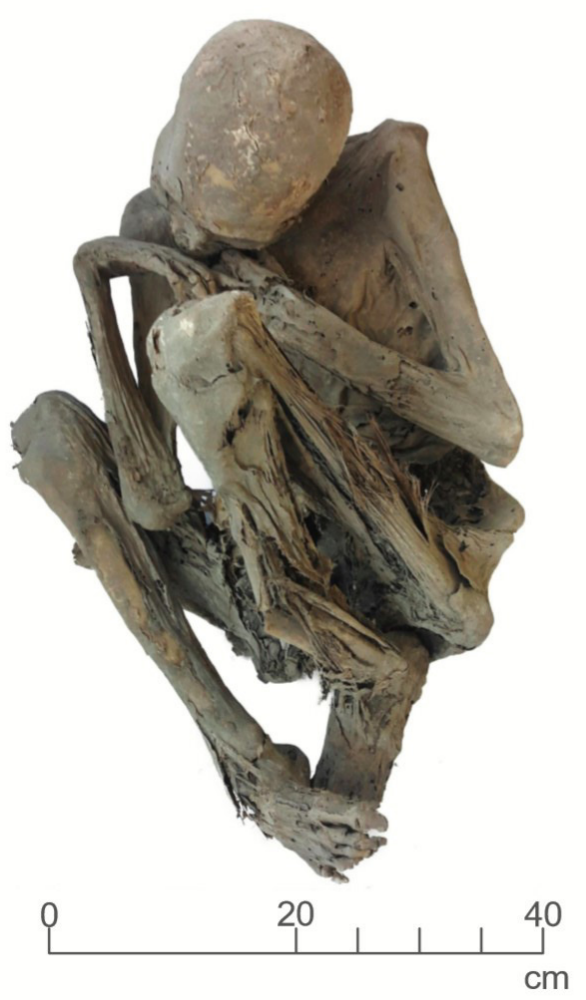

Figura. 1: individuo momificado en posición flexionado sentado. de los restos recuperados, ya que dejan evidencias importantes. Retomando la idea del entierro, Kaulicke (1997, pág. 25) menciona que "un contexto funerario normalmente se compone de tres elementos básicos: a) la estructura, b) el individuo y c) los objetos asociados". A partir de aquella premisa y de los materiales recuperados, se pretende identificar los restos en cada una de los elementos señalados.

Así, respecto al primer elemento, es decir, la estructura (espacio físico dónde se encontraban los individuos y los elementos asociados), fue completamente destruido durante la construcción de dicha obra. A pesar de ella, tenemos los relatos de algunos pobladores locales quienes indican que se trataba de dos estructuras arquitectónicas, donde una de ellas se encontraba en buen estado de conservación, mientras la otra se encontraba en mal estado de conservación. Estas se encontraban en la base de un afloramiento rocoso (comunicación personal con Hernán Ayala Huaracc y Saúl Conde). No se tiene mayor información respecto al tema. Cabe mencionar que el lugar donde se encontraba la estructura, no estaba asociado directamente a un asentamiento arqueológico, pero sí se encontraba a escasos metros del camino inca Pisco-Vilcashuamán. ¿Existe alguna relación con el camino? Asimismo, se tiene re- 
ferencias de la existencia de varios sitios arqueológicos en el área (Gentil Wayqu de Pirhuamarca, Qaqapaki, entre otros); por ella sería necesario determinar a partir de las prospecciones arqueológicas.

En relación al segundo elemento que es el individuo, se han registrado al menos 5 individuos de diferentes sexos y edades (desde restos de un infante hasta de individuos adultos) a partir de los análisis. Una de ellas presenta vestigios relevantes como evidencias de momificación en posición flexionado sentado, que demuestra una preparación para preservar el cuerpo del individuo (ver figura 1). Asimismo, al parecer, otro individuo más estuvo momificado ya que se registró piel seca adherida a los restos óseos (algunas de ellas parcialmente articuladas). Esto afianza que, en la región andina, desde los tiempos tempranos hasta la sociedad inca, buscaron proteger y preservar el cuerpo de los individuos muertos. Cada vez era diverso y más complejo en los tratamientos, cuidados y entierros (Valdez, 2014). Los demás individuos, fueron reconocidas a partir de los restos óseos, aunque son incompletos.

En cuanto al tercer y último elemento, es decir, los materiales asociados, se encontró regular cantidad de ella; aunque se desconoce la disposición de los materiales en relación al individuo y entre ellas. De todas ellas, resalta los materiales textiles como 2 unkus completos (con algunos desgarres y manchas), un posible bolso (chuspa) y fragmentos textiles de al menos de tres ejemplares. Generalmente, se tratan de textiles llanos elaborados de la fibra de camélido. Asimismo, se han registrados fragmentos de soguilla elaborados de la fibra vegetal y camélido. También, en el análisis se identificó 5 ejemplares de sandalias prehispánicas, las cuales presentan suelas de cuero, de una o dos pliegues; donde algunas de ellas muestran desgaste. De la misma forma, en algunos calzados se observa que, desde la suela se sujetan cuerdas trenzadas elaboradas de cabello humano, cuyo fin era sujetar el pie en la parte superior del calzado. Finalmente se encontró artefactos de mate, fragmentos de mate y un fragmento de cerámica no diagnóstica.

\section{CONSIDERACIONES FINALES}

Como se mencionó anteriormente, debido a la falta de contexto arqueológico, surgen muchos interrogantes, por ejemplo: ¿se trataba de un entierro múltiple? ¿A dónde estaban orientados los individuos? ¿cómo estaban dispuestos los elementos asociados en relación con los individuos y entre sí? ¿Habrá algún material no recuperado?, ¿de qué tipo de estructura funeraria se trataba?, entre otras. Además, estas nos abren a nuevas preguntas como: ¿los individuos fueron colocados al mismo tiempo? ¿pertenece a un grupo familiar?, ¿de qué época se trata? ¿a qué grupo cultural pertenece?, entre otras.

Pues, la identificación de los materiales disturbados de Chullkumayu, las cuales fueron expuestas de manera superficial en los párrafos anteriores, nos invitan a ha- 
cer mayor análisis sistemático de cada una de ellas; así entender las conductas, procesos sociales prehispánicas en el área. Asimismo, afianza a las referencias y escasas investigaciones arqueológicas desarrolladas en este espacio (Isbell, 2005) (Isbell, 2005) (Vivanco, 2005) (Muñoz Ruiz \& Núñez Espinoza, 2006) (Conde, 2014) (Yataco Capcha, Sánchez Tinco, \& León Canales , 2021). Esperemos que las futuras investigaciones nos permitan entender aún más los procesos sociales en la cuenca alta del río Pampas y la relación con otros espacios geográficos.

\section{REFERENCIAS BIBLIOGRÁFICAS}

Conde, N. (2014). Reconocimiento de Sitios Arqueológicos en la Comunidad de Canchacancha, Chuschi-Ayacucho. Ayacucho: Universidad Nacional de San Cristóbal de Huamanga.

Isbell, B. J. (2005). Para defendernos: Ecología y ritual en un pueblo andino. Cuzco: Centro de Estudios Regionales Andinos Bartolomé de Las Casas (CBC).

Kaulicke, P. (1997). La muerte en el antiguo Perú. Contextos y conceptos funerarios: una introducción. Boletín de Arqeuología PUCP, 7-54.

Muñoz Ruiz, U., \& Núñez Espinoza, O. (2006). Los Kanas de Quispillaccta. Historia de un pueblo quechua. Ayacucho: Ediciones Kana.

Valdez, E. (2014). ¿Pucullos? en las cuencas de los ríos Warpa, Urubamba y Mantaro, entre Ayacucho, Urubamba y Huancavelica, Perú. Arqueología y Sociedad 28, 199-216.

Vivanco, C. (2005). Condoray, un asentamiento Chanka en Canchacancha, Cangallo. Zanjas y murallas como estrategia de violencia prehispánica. Investigaciones, 129-139. Universidad Nacional de San Cristóbal de Huamanga,

Vivanco, C. (2014). El itenerario de colaboradores de Dr. J.C. Tello en la cuenca del río Pampas y Apurímac: Huancavelica, Apurímac y Cuzco. Revista Conchopata, 57-66.

Yataco Capcha, J. J., Sánchez Tinco, R., \& León Canales , E. (2021). Tecnología laminar del valle de Pampas, Ayacucho, Perú (ca. 0-900 d. C.). Arqueología y Sociedad (34), 9-32.

\section{SOBRE EL AUTOR}

\section{Juan Conde Vilca}

Estudiante de Arqueología de la Universidad Nacional de San Cristóbal de Huamanga, quién participó en diferentes proyectos arqueológicos. Actualmente viene realizando investigaciones arqueológicas en la cuenca alta del río Pampas. 